\title{
Space Alignment Based on Regularized Inversion Precoding in Cognitive Transmission
}

\author{
Rugui YAO ${ }^{1}$, Geng $L^{1}{ }^{1}$, Juan XU ${ }^{2}$, Ling $W A N G^{1}$, Zhaolin $Z H A N G{ }^{1}$ \\ ${ }^{1}$ School of Electronics and Information, Northwestern Polytechnical University, 710072 Xi' an, Shaanxi, China \\ ${ }^{2}$ School of Electronic and Control Engineering, Chang'an University, 710064 Xi' an, Shaanxi, China \\ yaorg@nwpu.edu.cn, 785462603@qq.com, xuj@mail.nwpu.edu.cn, lingwang@nwpu.edu.cn, z184@sina.com
}

\begin{abstract}
For a two-tier Multiple-Input Multiple-Output (MIMO) cognitive network with common receiver, the precoding matrix has a compact relationship with the capacity performance in the unlicensed secondary system. To increase the capacity of secondary system, an improved precoder based on the idea of regularized inversion for secondary transmitter is proposed. An iterative space alignment algorithm is also presented to ensure the Quality of Service $(Q o S)$ for primary system. The simulations reveal that, on the premise of achieving $Q o S$ for primary system, our proposed algorithm can get larger capacity in secondary system at low Signal-to-Noise Ratio (SNR), which proves the effectiveness of the algorithm.
\end{abstract}

\section{Keywords}

Cognitive network, Multiple-Input Multiple-Output (MIMO), space alignment, precoding, channel capacity

\section{Introduction}

With rapid development of wireless communications, the shortage of spectrum resources has become a bottleneck. To improve the spectrum efficiency, the state-of-the-art technique, cognitive radio [1], draws a lot of attention in recent years, where some primary users have priorities to use the spectrum bands and other secondary users can only transmit opportunistically without generating unacceptable interference to the primary ones. However, at high SNR, the available dimensions left by primary user are scarce, as stated in [2], [3], which results in low throughput of secondary users.

To address this problem, several approaches have been proposed. The novel concept of Interference Alignment (IA) is introduced to solve the interference in licensed network caused by cognitive network [4]. In [5], [6], a transmitted precoding matrix is designed to align the signal of cognitive user into the null space of channel matrix of primary system, and a post-processing matrix at cognitive receiver is also designed to whiten the interference from primary system. By this means, the two systems can simultaneously access the spectrum. In [7], multiple cognitive users are considered as an extension of the work in [5], [6]. To deal with the scarcity of transmitted dimensions for cognitive users at high SNR, a threshold water-filling power allocation algorithm is ingeniously designed. By the modified threshold, some transmitted dimensions of primary system are released without damaging its QoS. With the emerging dimensions, the precoding matrices for both primary transmitter and cognitive transmitter and the post-processing matrix for the common receiver are also designed in [8]. Notice that, in [8], the design of precoding matrix for cognitive transmitter does not consider the normalization of power, which destroys the transmitted power constraint for the cognitive system.

In this paper, we extend the work in [8] with considering the power normalization for precoder. An improved precoder design and space alignment scheme based on regularized inversion [9], [10] are proposed. The proposed method can achieve capacity improvement for secondary system at low SNR.

The rest of the paper is organized as follows. In Sec. 2, we describe the system model. In Sec. 3, we propose the improved precoder design and the space alignment scheme after analyzing the impact of power normalization. Section 4 presents some numerical results to validate the effectiveness of our proposed method. Finally, Sec. 5 concludes this paper.

\section{System Model}

As shown in Fig. 1, the primary transmitter, P, and the cognitive transmitter, $S$, share the same spectrum, and send their own information simultaneously to a common receiver, R. P has the priority to use the spectrum and S only opportunistically utilizes the dimensions left by the primary transmitter without interfering the reception of primary signal. All transmitters, $\mathrm{P}$ and $\mathrm{S}$, and the common receiver, $\mathrm{R}$, are equipped with $M(M>1)$ antennas.

The received signal vector, $\mathbf{y} \in C^{M \times 1}$, at $\mathrm{R}$ can be represented as

$$
\mathbf{y}=\mathbf{H}_{\mathrm{RP}} \mathbf{G}_{\mathrm{P}} \mathbf{x}_{\mathrm{P}}+\mathbf{H}_{\mathrm{RS}} \mathbf{G}_{\mathrm{S}} \mathbf{x}_{\mathrm{S}}+\mathbf{z},
$$




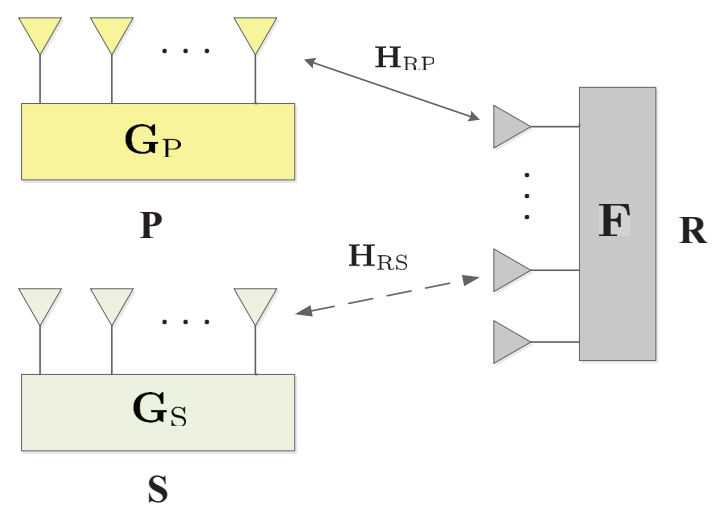

Fig. 1. System model of the two-tier cognitive network.

where $\mathbf{x}_{\mathrm{P}} \in C^{M \times 1}$ and $\mathbf{x}_{\mathrm{S}} \in C^{M \times 1}$ denote the symbol vectors transmitted by $\mathrm{P}$ and $\mathrm{S}$; the transmission of $\mathrm{P}$ and $\mathrm{S}$ is subjected to a total power constraint, $P_{d}$, i.e., Trace $\left\{\mathbf{x}_{\mathrm{P}} \mathbf{x}_{\mathrm{P}}^{\mathrm{H}}\right\} \leq P_{d}$ and Trace $\left\{\mathbf{x}_{\mathrm{S}} \mathbf{x}_{\mathrm{S}}^{\mathrm{H}}\right\} \leq P_{d} ; \mathbf{G}_{\mathrm{P}} \in C^{M \times M}$ and $\mathbf{G}_{\mathrm{S}} \in C^{M \times M}$ represent the precoding matrices of transmitter $\mathrm{P}$ and $\mathrm{S}$, which are orthonormal with respect to their columns and satisfy $\operatorname{Trace}\left\{\mathbf{G}_{\mathrm{P}} \mathbf{G}_{\mathrm{P}}^{\mathrm{H}}\right\}=\operatorname{Trace}\left\{\mathbf{G}_{\mathrm{S}} \mathbf{G}_{\mathrm{S}}^{\mathrm{H}}\right\}=M ; \mathbf{H}_{\mathrm{RP}} \in C^{M \times M}$ and $\mathbf{H}_{\mathrm{RS}} \in C^{M \times M}$ denote the channel matrices from $\mathrm{P}$ and $\mathrm{S}$ to $\mathrm{R}$; each element in $\mathbf{H}_{\mathrm{RP}}$ and $\mathbf{H}_{\mathrm{RS}}$ is $\mathcal{C} \mathcal{N}(0,1)$ distributed and independent with each other; $\mathbf{z} \in C^{M \times 1}$ indicates the Zero Mean Circular Symmetric Complex Gaussian Noise (ZMCSCGN) and is $\mathcal{C} \mathcal{N}\left(\mathbf{0}, \sigma_{n}^{2} \mathbf{I}_{M}\right)$ distributed. At the common receiver, $\mathrm{R}$, we adopt post-processing matrix, $\mathbf{F} \in C^{M \times M}$, to detect the signal from $\mathrm{P}$ and $\mathrm{S}, \widehat{\mathbf{x}}_{\mathrm{P}}$ and $\widehat{\mathbf{x}}_{\mathrm{S}}$.

We further assume the channels of the primary and cognitive networks experience flat slow fading. Note that some Channel State Information's (CSIs) are required for the design of precoding and post-processing matrices. We assume $\mathrm{P}$ and $\mathrm{R}$ only acquire the perfect $\mathbf{H}_{\mathrm{RP}}$ of the primary system, and $\mathbf{S}$ can get the whole perfect CSIs, $\mathbf{H}_{\mathrm{RS}}$ and $\mathbf{H}_{\mathrm{RP}}$. Actually, the CSIs can be acquired by the channel reciprocity in Time-Division Duplexing (TDD) communications [11] and CSI exchange techniques in Frequency-Division Duplexing (FDD) communications [12].

\section{Space Alignment Scheme Based on Regularized Inversion}

In this section, we first review the precoder design of primary system. And then we propose an improved precoder design for secondary system and space alignment based on regularized inversion as an important extension of the work in [8].

\subsection{Review of Primary System Design}

To maximize the throughput of primary system, we should take Singular Value Decomposition (SVD) to the channel matrix, $\mathbf{H}_{\mathrm{RP}}$, to decouple the channel and adopt Water-filling Power Allocation (WPA) algorithm to optimally allocate different power on the decoupled channels.
Denote the SVD of $\mathbf{H}_{\mathrm{RP}}$ as

$$
\mathbf{H}_{\mathrm{RP}}=\mathbf{U}_{\mathrm{P}} \Lambda_{\mathrm{P}} \mathbf{V}_{\mathrm{P}}^{\mathrm{H}},
$$

where $\mathbf{U}_{\mathrm{P}} \in C^{M \times M}$ and $\mathbf{V}_{\mathrm{P}} \in C^{M \times M}$ are unitary; and $\Lambda_{\mathrm{P}}=$ $\operatorname{diag}\left\{\lambda_{\mathrm{P} 1}, \lambda_{\mathrm{P} 2}, \cdots, \lambda_{\mathrm{P} M}\right\} \in C^{M \times M}$ is composed of all the singular values of $\mathbf{H}_{\mathrm{RP}}$ in descending order. And then the precoding and post-processing matrices for primary system can be designed as

$$
\mathbf{G}_{\mathrm{P}}=\mathbf{V}_{\mathrm{P}} \text { and } \mathbf{F}=\mathbf{U}_{\mathrm{P}}^{\mathrm{H}}
$$

With $\mathbf{G}_{\mathrm{P}}$ and $\mathbf{F}$, the detected symbols of primary system at receiver $\mathrm{R}, \widehat{\mathbf{x}}_{\mathrm{P}} \in C^{M \times 1}$, can be represented as

$$
\begin{aligned}
\widehat{\mathbf{x}}_{\mathrm{P}} & =\mathbf{F y}=\mathbf{U}_{\mathrm{P}}^{\mathrm{H}} \mathbf{y} \\
& =\mathbf{U}_{\mathrm{P}}^{\mathrm{H}}\left[\left(\mathbf{U}_{\mathrm{P}} \Lambda_{\mathrm{P}} \mathbf{V}_{\mathrm{P}}^{\mathrm{H}}\right) \mathbf{V}^{\mathrm{P}} \mathbf{x}_{\mathrm{P}}+\mathbf{H}_{\mathrm{RS}} \mathbf{G}_{\mathrm{S}} \mathbf{x}_{\mathrm{S}}+\mathbf{z}\right] \\
& =\Lambda_{\mathrm{P}} \mathbf{x}_{\mathrm{P}}+\mathbf{U}_{\mathrm{P}}^{\mathrm{H}} \mathbf{H}_{\mathrm{RS}} \mathbf{G}_{\mathrm{S}} \mathbf{x}_{\mathrm{S}}+\mathbf{z}^{\prime},
\end{aligned}
$$

where $\mathbf{z}^{\prime}=\mathbf{U}_{\mathrm{P}}^{\mathrm{H}} \mathbf{z}$ is still $\mathcal{C} \mathcal{N}\left(\mathbf{0}, \sigma_{n}^{2} \mathbf{I}_{M}\right)$ distributed due to the unitary property of $\mathbf{U}_{\mathrm{P}}^{\mathrm{H}}$. The second item in (4) denotes the interference from cognitive transmitter $\mathrm{S}$ to the reception of $\mathbf{x}_{\mathrm{P}}$. We first focus on capacity maximization for primary system ignoring the interference from S. In Sec. 3.2, the precoder for cognitive transmitter $\mathrm{S}$ will be developed in detail to avoid this interference. The diagonal elements of $\Lambda_{P}$ in (4) represent the gain of the equivalent decoupled channel. According to $\Lambda_{\mathrm{P}}$, WPA algorithm can be implemented to further maximize the capacity of primary system.

Denote $\mathbf{P}_{\mathrm{P}}=\operatorname{diag}\left(P_{\mathrm{P} 1}, P_{\mathrm{P} 2}, \cdots, P_{\mathrm{P} M}\right) \in C^{M \times M}$ and $\mathbf{s}_{\mathrm{P}} \in$ $C^{M \times 1}$ be the power allocation matrix and the transmitted symbol with unitary power for primary system, respectively. Thus, we have $\mathbf{x}_{\mathrm{P}}=\mathbf{P}_{\mathrm{P}} \mathbf{S}_{\mathrm{p}}$. With WPA algorithm, the power allocation matrix, $\mathbf{P}_{\mathrm{P}}$, is derived as follows [13]

$$
\begin{aligned}
& P_{\mathrm{P} k}= \begin{cases}\mu-\frac{M \sigma_{n}^{2}}{P_{d} \lambda_{\mathrm{P} k}^{2}} & \text { if }\left(\mu-\frac{M \sigma_{n}^{2}}{P_{d} \lambda_{\mathrm{Pk}}^{2}}\right) \geq 0, \\
0 & \text { else, }\end{cases} \\
& k=1,2, \cdots, M,
\end{aligned}
$$

where the constant $\mu$ represents the horizontal line of power for WPA algorithm. Note that the kth dimension in $\mathbf{S}_{\mathrm{P}}$ cannot be used to transmit symbols of primary system if $P_{\mathrm{P} k}=0$.

As stated in [8], at high SNR, all $P_{\mathbf{P} k} \mathrm{~S}$ are positive and large capacity is then achieved. However, for two-tier networks, the dimensions left for cognitive system is few or even zero. Therefore, cognitive network cannot transmit its symbols or can work with low throughput. In [8] and [14], a modified WPA algorithm with a threshold is proposed to solve this issue. By introducing a threshold, primary system can release some eigen-directions with relative low channel gains to cognitive system while guaranteeing the QoS requirement for primary system. The power allocation matrix with modified WPA algorithm, $\mathbf{P}_{\mathrm{P}}\left(P_{t h}\right)=$ $\operatorname{diag}\left(P_{\mathrm{P} 1}\left(P_{t h}\right), P_{\mathrm{P} 2}\left(P_{t h}\right), \cdots, P_{\mathrm{P} M}\left(P_{t h}\right)\right) \in C^{M \times M}$, can be computed as 


$$
\begin{aligned}
& P_{\mathrm{P} k}\left(P_{t h}\right)= \begin{cases}\mu-\frac{M \sigma_{n}^{2}}{P_{d} \lambda_{\mathrm{P} k}^{2}} & \text { if }\left(\mu-\frac{M \sigma_{n}^{2}}{P_{d} \lambda_{\mathrm{Pk}}^{2}}\right) \geq P_{t h}, \\
0 & \text { else, }\end{cases} \\
& k=1,2, \cdots, M,
\end{aligned}
$$

where the constant threshold, $P_{t h} \in[0, P d)$, depends on the $\mathrm{QoS}$ requirement of primary system. In this case, extra diagonal elements of $\mathbf{P}_{\mathrm{P}}\left(P_{t h}\right)$ are set to be 0 , which means more dimensions are squeezed for transmission of cognitive system. And the corresponding capacity can be denoted as

$$
C\left(P_{t h}\right)=\log _{2} \operatorname{det}\left(\mathbf{I}_{M}+\mathbf{P}_{\mathrm{P}}\left(P_{t h}\right) \Lambda_{\mathrm{P}} \Lambda_{\mathrm{P}}^{\mathrm{H}}\right) .
$$

Generally, the required QoS is denoted in terms of capacity as $C_{\alpha}^{r}=\alpha C_{0}, \alpha \in(0,1]$, where the maximal capacity $C_{0}$ can be achieved by (5) or by (6) with $P_{t h}=0$ [8]. For a given $\alpha$, we always select the maximum of $P_{t h}$ to make $C\left(P_{t h}\right) \geq \alpha C_{0}$.

\subsection{Precoder Design for Secondary System}

From (4), to avoid interference to primary system, the precoding matrix for cognitive system must satisfy the following condition,

$$
\mathbf{U}_{\mathrm{P}}^{\mathrm{H}} \mathbf{H}_{\mathrm{RS}} \mathbf{G}_{\mathrm{S}}=\beta \overline{\mathbf{P}}^{1 / 2}
$$

where normalization factor $\beta$ is to guarantee the constraint for $\mathbf{G}_{\mathrm{S}}$, Trace $\left\{\mathbf{G}_{\mathrm{S}} \mathbf{G}_{\mathrm{S}}^{\mathrm{H}}\right\}=M$, and the matrix $\overline{\mathbf{P}}=$ $\operatorname{diag}\left(\bar{P}_{1}, \bar{P}_{2}, \cdots, \bar{P}_{M}\right) \in \mathcal{C}^{M \times M}$ can be computed as

$$
\begin{gathered}
\bar{P}_{k}= \begin{cases}1 & \text { if } P_{\mathrm{P} k}\left(P_{t h}\right)=0, \\
0 & \text { else, }\end{cases} \\
k=1,2, \cdots, M .
\end{gathered}
$$

Due to the eigen-direction releasing strategy in (6), we have $\overline{\mathbf{P}}=\operatorname{diag}(0, \cdots, 0,1, \cdots, 1)$. And $m=\sum_{k=1}^{M} \bar{P}_{k}$ denotes the total degrees of freedom left by primary system, which can be used by cognitive system to transmit their symbols.

Without loss of generality, $\mathbf{H}_{\mathrm{RS}}$ is assumed to be full ranked. And thus, the precoding matrix for cognitive system, can be derived from (8) as

$$
\mathbf{G}_{\mathrm{S}}=\beta \mathbf{H}_{\mathrm{RS}}^{-1} \mathbf{U}_{\mathrm{P}} \overline{\mathbf{P}}^{1 / 2} \text {. }
$$

Denote $\mathbf{P}_{\mathrm{S}}=\operatorname{diag}\left(P_{\mathrm{S} 1}, P_{\mathrm{S} 2}, \cdots, P_{\mathrm{S} M}\right) \in C^{M \times M}$ be the power allocation matrix for cognitive network. With (8) and (10), the average capacity for cognitive system can be further computed as

$$
\begin{aligned}
C_{s} & =\max _{\mathbf{P}_{\mathrm{S}}} \log _{2} \operatorname{det}\left(\mathbf{I}_{M}+\frac{\beta^{2}}{\sigma_{n}^{2}} \mathbf{U}_{\mathrm{P}}^{\mathrm{H}} \mathbf{H}_{\mathrm{RS}} \mathbf{G}_{\mathrm{S}} \mathbf{P}_{\mathrm{S}} \mathbf{G}_{\mathrm{S}}^{\mathrm{H}} \mathbf{H}_{\mathrm{RS}}^{\mathrm{H}} \mathbf{U}_{\mathrm{P}}\right) \\
& =\max _{\mathbf{P}_{\mathrm{S}}} \log _{2} \operatorname{det}\left(\left(\mathbf{I}_{M}+\frac{\beta^{2}}{\sigma_{n}^{2}} \overline{\mathbf{P}} \mathbf{P}_{\mathrm{S}}\right)\right. \\
& =\max _{\mathbf{P}_{\mathrm{S}}} \sum_{k=1}^{M} \log _{2}\left(1+\frac{\beta^{2}}{\sigma_{n}^{2}} \bar{P}_{k} P_{\mathrm{S} k}\right) \\
& =m \log _{2}\left(1+\frac{\beta^{2} P_{d}}{m \sigma_{n}^{2}}\right)
\end{aligned}
$$

Note that, the uniform power allocation with $P_{\mathrm{S} k}=\frac{P_{d}}{m}$ is applied to achieve the maximum channel capacity due to the arithmetic-geometric inequality [15]. From (11), it is observed that the upper bound of channel capacity of secondary system largely depends on $\beta$. Thus, increasing $\beta$ becomes an effective way to improve capacity.

With SVD, $\mathbf{H}_{\mathrm{RS}}$ is represented as $\mathbf{H}_{\mathrm{RS}}=\mathbf{U}_{\mathrm{S}} \Lambda_{\mathrm{S}} \mathbf{V}_{\mathrm{S}}^{\mathrm{H}}$, where $\mathbf{U}_{\mathrm{S}} \in C^{M \times M}$ and $\mathbf{V}_{\mathrm{S}} \in C^{M \times M}$ are unitary; and therefore, $\mathbf{H}_{\mathrm{RS}}^{-1}=\mathbf{V}_{\mathrm{S}} \Lambda_{\mathrm{S}}^{-1} \mathbf{U}_{\mathrm{S}}^{\mathrm{H}} \cdot \Lambda_{\mathrm{S}}$ and $\Lambda_{\mathrm{S}}^{-1}$ are defined as

$$
\begin{gathered}
\Lambda_{\mathrm{S}}=\operatorname{diag}\left\{\lambda_{\mathrm{S} 1}, \lambda_{\mathrm{S} 2}, \cdots, \lambda_{\mathrm{S} M}\right\}, \\
\Lambda_{\mathrm{S}}^{-1}=\operatorname{diag}\left\{\frac{1}{\lambda_{\mathrm{S} 1}}, \frac{1}{\lambda_{\mathrm{S} 2}}, \cdots, \frac{1}{\lambda_{\mathrm{S} M}}\right\},
\end{gathered}
$$

where $\lambda_{\mathrm{S} 1} \geq \lambda_{\mathrm{S} 2} \cdots \geq \lambda_{\mathrm{S} M}$.

Considering (10), we can further calculate that

$$
\begin{aligned}
\operatorname{Trace}\left\{\mathbf{G}_{\mathrm{S}} \mathbf{G}_{\mathrm{S}}^{\mathrm{H}}\right\} & =\operatorname{Trace}\left\{\beta^{2} \mathbf{H}_{\mathrm{RS}}^{-1} \mathbf{U}_{\mathrm{P}} \overline{\mathbf{P}} \mathbf{U}_{\mathrm{P}}^{\mathrm{H}}\left(\mathbf{H}_{\mathrm{RS}}^{-1}\right)^{\mathrm{H}}\right\} \\
& =\beta^{2} \sum_{k=M-m+1}^{M}\left(\frac{1}{\lambda_{\mathrm{S} k}}\right)^{2} \\
& =M .
\end{aligned}
$$

From (14), if $\mathbf{H}_{\mathrm{RS}}$ is an ill-conditioned matrix, especially at low SNR, that is, some singular values, $\lambda_{\mathrm{S} k}$, are very small, then $\sum_{k=M-m+1}^{M}\left(\frac{1}{\lambda_{\mathrm{sk}}}\right)^{2}$ will be a large number. In this case, to satisfy the constraint $\operatorname{Trace}\left\{\mathbf{G}_{\mathrm{S}} \mathbf{G}_{\mathrm{S}}^{\mathrm{H}}\right\}=M, \beta$ will be a small number, which leads to the decline of capacity performance for secondary system from (11). To solve this problem, a concept of regularized inversion [10] is introduced to calculate the inverse matrix $\mathbf{H}_{\mathrm{RS}}^{-1}$ as

$$
\widetilde{\mathbf{H}}_{\mathrm{RS}}^{-1}=\mathbf{H}_{\mathrm{RS}}^{\mathrm{H}}\left(\mathbf{H}_{\mathrm{RS}} \mathbf{H}_{\mathrm{RS}}^{\mathrm{H}}+\varepsilon^{2} \mathbf{I}_{\mathrm{M}}\right)^{-1},
$$

where $\varepsilon^{2}$, with initial value $\frac{M \sigma_{n}^{2}}{P_{d}}[9,10]$, is an introduced interference factor. In the following subsection, we will detailedly present the selection strategy of $\varepsilon^{2}$. With (15), the singular-value matrix $\widetilde{\Lambda}_{\mathrm{S}}^{-1}$ of $\widetilde{\mathbf{H}}_{\mathrm{RS}}^{-1}$ and the improved precoding matrix for secondary system can be modified as follows

$$
\begin{gathered}
\widetilde{\Lambda}_{\mathrm{S}}^{-1}=\operatorname{diag}\left\{\frac{\lambda_{\mathrm{S} 1}}{\lambda_{\mathrm{S} 1}^{2}+\varepsilon^{2}}, \frac{\lambda_{\mathrm{S} 2}}{\lambda_{\mathrm{S} 2}^{2}+\varepsilon^{2}}, \cdots, \frac{\lambda_{\mathrm{S} M}}{\lambda_{\mathrm{S} M}^{2}+\varepsilon^{2}}\right\}, \\
\widetilde{\mathbf{G}}_{\mathrm{S}}=\widetilde{\beta} \widetilde{\mathbf{H}}_{\mathrm{RS}}^{-1} \mathbf{U}_{\mathrm{P}} \overline{\mathbf{P}}^{1 / 2},
\end{gathered}
$$

where $\widetilde{\beta}$ can be obtained by replacing $\frac{1}{\lambda_{s k}}$ in (14) by $\frac{\lambda_{S k}}{\lambda_{S k}^{2}+\varepsilon^{2}}$ in (16).

At low SNR, as $\varepsilon^{2}=\frac{M \sigma_{n}^{2}}{P_{d}}$, we have $\frac{\lambda_{\mathrm{Sk}}}{\lambda_{\mathrm{Sk}}^{2}+\varepsilon^{2}}<\frac{1}{\lambda_{\mathrm{Sk}}}$. Further from (14), the decreasing of $\sum_{k=M-m+1}^{M}\left(\frac{\lambda_{S k}}{\lambda_{\mathrm{S} k}+\varepsilon^{2}}\right)^{2}$ will 
lead to the increase of $\widetilde{\beta}$. And thus, the capacity of secondary system is improved from (11). At high SNR, the interference factor $\varepsilon^{2}$ is small enough to be ignored and the capacity of the two-tier system can remain unchanged. Besides, it should be noted that with new precoding matrix signals sending by the $\mathrm{S}$ will introduce certain interference to the primary system, so the selection of $\varepsilon^{2}$ should compromise between the QoS requirement for primary system and the capacity improvement for secondary system. In the following part, an effective SA scheme is proposed to maximize the channel capacity of the secondary system while guaranteeing the QoS requirement of primary system.

\subsection{Iterative Space Alignment Scheme}

With the precoder based on regularized inversion, the received signals for $\mathrm{P}$ at $\mathrm{R}$ will include the crosstalk interference from $\mathrm{S}$. The initial value of $\frac{M \sigma_{n}^{2}}{P_{d}}$ for $\varepsilon^{2}$ can only maximize the Signal-to-Interference-plus-Noise Ratio (SINR) and thus the capacity for secondary system [10]; however, it is with high priority to ensure the QoS requirement of primary system. When the interference introduced from secondary system is too large, reducing $\varepsilon^{2}$ is an effective way to increase the capacity of primary system, which will be also demonstrated by the numerical results in Sec. 4. To guarantee the QoS requirement of primary system and maximize the capacity of secondary system, a simple iterative algorithm is developed.

1) $P$ and $R$ acquire the perfect $\mathbf{H}_{R P}$ of the primary system; and thus, both of them can achieve the water-filling power threshold $P_{t h}$ with the QoS requirement of $\alpha C_{0}$, and also the precoding matrix $\mathbf{G}_{\mathrm{P}}$, post-processing matrix $\mathbf{F}$, the power allocation matrix $\mathbf{P}_{\mathbf{P}}$ from (2), (3) and (6).

2) Assuming $S$ has the whole perfect CSIs, it can get not only all the calculated results in step 1) but also the improved precoding matrix $\widetilde{\mathbf{G}}_{\mathrm{S}}$ with (17). Furthermore, the initial capacity of primary system with $\varepsilon^{2}=\frac{M \sigma_{n}^{2}}{P_{d}}$ can be computed as

$$
C_{\mathrm{P}_{0}}=\sum_{k=1}^{M-m} \log _{2}\left(1+\frac{S(k)}{\sigma_{n}^{2}+J(k)}\right),
$$

where $S(k)$ and $J(k)$ represents the desired signal power and the "cross-introduced" interference power of $k$ th symbol, respectively.

3) At transmitter $\mathrm{S}$, compare $C_{\mathrm{P} i}$ with $\alpha C_{0}$ where $i=$ $0,1, \cdots$ denotes the iterative number. If $C_{\mathrm{P} i}<\alpha C_{0}$, that is, the QoS requirement is not satisfied, then reduce $\varepsilon^{2}$ by a fix small step, $\varepsilon^{2}=\varepsilon^{2}-\frac{M \sigma_{n}^{2}}{P_{d} K}$, where $K$ denotes the maximum number of iterations; and go back to step 2). Otherwise, if $C_{\mathrm{P} i} \geq \alpha C_{0}$, stop the iteration and optimal SA scheme is achieved.

In this scheme, the QoS requirement of the primary system is guaranteed through the adaptive adjustment of the introduced interference from secondary system. Besides, the throughput of the secondary system can also be improved significantly.

\section{Numerical Results}

In this section, we present some numerical results to validate the effectiveness of the proposed algorithm. The transmitters $\mathrm{P}$ and $\mathrm{S}$ and receiver $\mathrm{R}$ are all equipped with $M=8$ antennas. The transmitted power for single antenna is normalized and thus $P_{d}=8 . \alpha$ is set to be 0.9 to ensure high QoS for primary system.

Figure 2 shows the capacities for both primary system and secondary system versus $\varepsilon^{2}$ for $\mathrm{SNR}=0 \mathrm{~dB}$ and $\mathrm{SNR}=$ $5 \mathrm{~dB}$. Here, $\varepsilon^{2}$ is normalized by $\frac{M \sigma_{n}^{2}}{P_{d}}$. From Fig. 2 , it can be observed that, with the decline of $\varepsilon^{2}$, the capacity of primary system is monotonely increasing while the capacity of primary system is monotonely decreasing. Specifically, when $\varepsilon^{2}=0$, there is no "crosstalk" interference from $S$ to the received signal for primary system. With the introduction of $\varepsilon^{2}$, the secondary system can achieve a large improvement. To ensure the QoS requirement of primary system, $\varepsilon^{2}$ should be reduced to a proper value. Even in that case, the secondary system can still achieve a large capacity improvement as shown in Fig. 2.

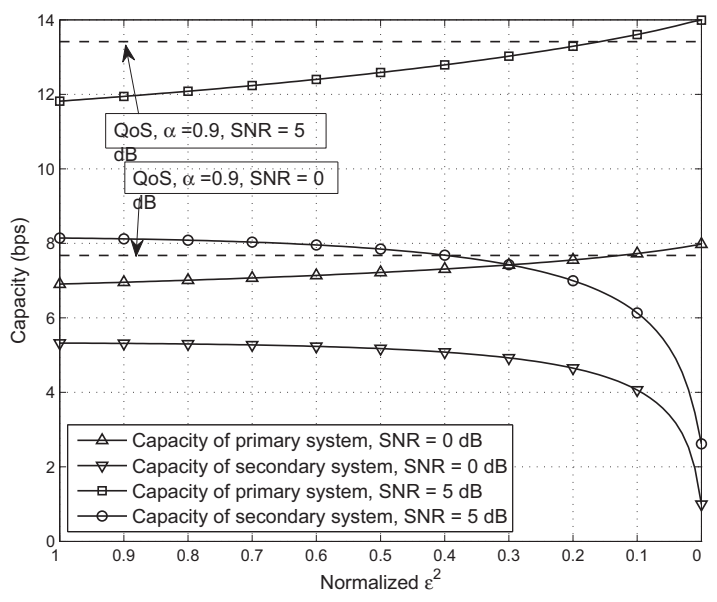

Fig. 2. Capacities for both primary system and secondary system versus normalized $\varepsilon^{2}$ for $\mathrm{SNR}=0 \mathrm{~dB}$ and $\mathrm{SNR}=$ $5 \mathrm{~dB}$.

Figure 3 compares the capacities of both primary system and secondary system when the algorithm in [8] and the proposed algorithm are adopted. From Fig. 3, for the capacity performance of secondary system, our proposed algorithm outperforms that in [8], especially at low SNR. Also we should notice that, in the secondary system, the capacity of our proposed algorithm is little worse than that with when the initial value of $\varepsilon^{2}$ is applied. As stated in Sec. 3.3, the initial value of $\frac{M \sigma_{n}^{2}}{P_{d}}$ for $\varepsilon^{2}$ aims to maximize the SINR and thus the largest capacity for secondary system. However, this procedure will generate interference to primary system, even destroy the QoS requirement of primary system. Therefore, 


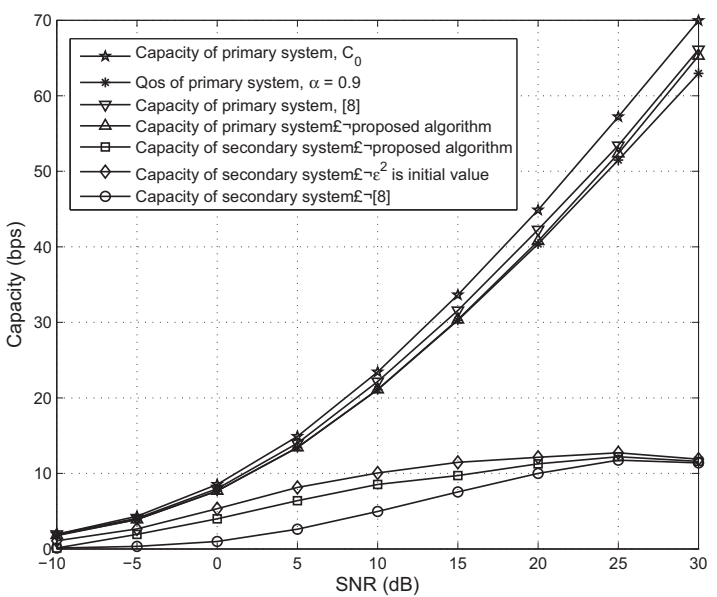

Fig. 3. Capacities for both primary and secondary systems with different algorithms.

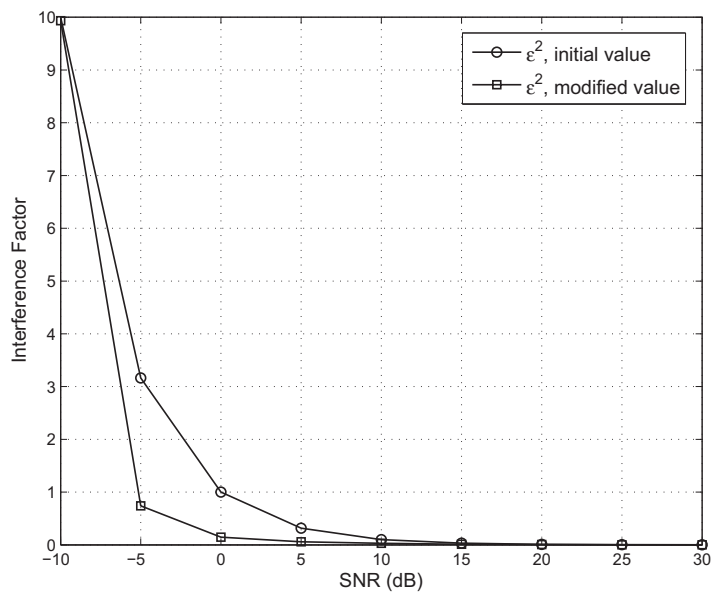

Fig. 4. Adjustment of $\varepsilon^{2}$ at different SNR.

the interference to primary system should be deceased by reducing $\varepsilon^{2}$. Figure 4 illustrates the modification procedure of $\varepsilon^{2}$. By this means, the QoS requirement of primary system is satisfied while some capacity improvement is still achieved.

For primary system, our proposed algorithm and that in [8] both meet the QoS requirements. However, there exists some gaps between the two algorithms. It attributes to the following two causes: a). The capacity of the primary system is achieved by threshold-based WPA algorithm, which is essential to select minimum available spatial dimensions. In this case, the primary system usually obtains larger capacity than the QoS requirement, especially at high SNR. This will lead to some performance redundancy for the primary system. b). The improved algorithm in this paper can adaptively adjust the amount of the interference to make the capacity of the primary system be closer to the QoS requirement. At high SNR, both algorithms achieve similar throughput for both primary system and secondary system because $\varepsilon^{2}$ is too small to be ignored.

\section{Conclusion}

Considering a special network with a primary transmitter, a secondary transmitter and a common receiver, we propose a regularized inversion based precoder design and an iterative space alignment scheme. By adaptively adjusting the introduced interference from secondary system to primary system, the proposed algorithm can maximize the capacity of secondary system while guaranteeing the QoS requirement of primary system. Numerical results demonstrate the capacity improvement of secondary system, especially at low SNR.

\section{Acknowledgments}

This work was supported in part by the National Natural Science Foundation of China (No. 61271416 and 61301093), the Aerospace support fund of China (No. 2013HT-XGD) and Natural Science Basic Research Plan in Shaanxi Province of China (No. 2014JM2-6094).

\section{References}

[1] HONG, X. M., CHEN, Z. M., WANG, C. X., et al. Cognitive radio networks. IEEE Vehicular Technology Magazine, 2009, vol. 4, no. 4, p. 76-84. DOI: 10.1109/MVT.2009.934672

[2] ZHANG, R., LIANG, Y.-C. Exploiting multi-antennas for opportunistic spectrum sharing in cognitive radio networks. IEEE Journal of Selected Topics in Signal Processing, 2008, vol. 2, no. 1, p. 88-102. DOI: 10.1109/JSTSP.2007.914894

[3] PHAN, K. T., VOROBYOV, S. A., SIDIROPOULOS, N. D., et al. Spectrum sharing in wireless networks via QoS-aware secondary multicast beamforming. IEEE Transactions on Signal Processing, 2009, vol. 57, no. 6, p. 2323-2335. DOI: 10.1109/TSP.2009.2016262

[4] CADAMBE, V. R., JAFAR, S. A. Interference alignment and degrees of freedom for the K-uer interference channel. IEEE Transactions on Information Theory, 2008, vol. 54, no. 8, p. 3425-3441. DOI: 10.1109/TIT.2008.926344

[5] PERlaZA, S. M., DEBBAH, M., LASAUlCE, S., et al. Opportunistic interference alignment in MIMO interference channels. In Proceedings of IEEE 19th International Symposium on Personal, Indoor and Mobile Radio Communications (PIMRC). Cannes (France), 2008, p. 2484-2488. DOI: 10.1109/PIMRC.2008.4699872

[6] PERlaza, S. M., FAWAZ, N., LASAUlCE, S., et al. From spectrum pooling to space pooling: opportunistic interference alignment in MIMO cognitive networks. IEEE Transactions on Signal Processing, 2010, vol. 58, no. 7, p. 3728-3741. DOI: 10.1109/TSP.2010.2046084

[7] HUPPERT, C. Opportunistic resource allocation in MIMO cognitive systems with multiple users. In Proceedings of 2011 International ITG Workshop on Smart Antennas (WSA). Aachen (Germany), 2011, p. 1-6. DOI: 10.1109/WSA.2011.5741931 
[8] KRIKIDIS, I. Space alignment for cognitive transmission in MIMO uplink channels. EURASIP Journal on Wireless Communications and Networking, 2010, vol. 2010. DOI: 10.1155/2010/465157

[9] MASO, M., CARDOSO, L. S., DEBBAH, M., et al. Cognitive orthogonal precoder for two-tiered networks deployment. IEEE Journal on Selected Areas in Communications, 2011, vol. 31, no. 11, p. 2338-2348. DOI: 10.1109/JSAC.2013.131108

[10] PEEL, C. B., HOCHWALD, B. M., SWINDLEHURST, A. L. A vector-perturbation technique for near-capacity multiantenna multiuser communication - Part I: channel inversion and regularization. IEEE Transactions on Communications, 2005, vol. 53, no. 1, p. 195-202. DOI: 10.1109/TCOMM.2004.840638

[11] MASO, M., CARDOSO, L. S., DEBBAH, M., et al. Channel estimation impact for LTE small cells based on MU-VFDM. In Proceedings of 2012 IEEE Wireless Communications and Networking Conference (WCNC). Shanghai (China), 2012, p. 2560-2565. DOI: 10.1109/WCNC.2012.6214230

[12] PANTISANO, F., BENNIS, M., SAAD, W., et al. Improving macrocell-small cell coexistence through adaptive interference draining. IEEE Transactions on Wireless Communications, 2013, vol. 13, no. 2, p. 942-955. DOI: 10.1109/TWC.2013.120613.130617

[13] CHO, Y. S., KIM, J., YANG, W. Y., et al. MIMO-OFDM Wireless Communications With MATLAB. 1st ed. Singapore (Singapore): John Wiley \& Sons (Asia) Pte Ltd, 2010. ISBN: 9780070825617

[14] BAI, X. J. Space alignment for cognitive transmission with multiple primary users. Electronics Letters, 2013, vol. 49, no. 25, p. 1619-1620. DOI: 10.1049/el.2013.2524

[15] JEFFREY, A., ZWILLINGER, D. Table of Integrals, Series, and Products. 7th ed. Salt Lake City (USA): Academic Press, 2007. ISBN: 9780123736376

\section{About the Authors ...}

Rugui YAO (corresponding author) was born in Anhui, China, in 1980. He received the BS, MS and Ph.D degrees all in the School of Electronics and Information from Northwestern Polytechnical University (NPU), Xi'an, China, in 2002, 2005 and 2007, respectively. He worked as a postdoctoral fellow at NPU from 2007 to 2009. After that, he joined this school and became an associate professor in 2010. In the year of 2013, he joined ITP Lab of Georgia Tech as a visiting scholar. He has worked in the area of cognitive radio networks, channel coding, OFDM transmission and spread spectrum systems.

Geng LI was born in Hebei, China, in 1990. He received his bachelor degree in the School of Electronics and Information from Northwestern Polytechnical University (NPU), Xi'an, China, in 2012 and currently he is pursuing his MS degree at NPU. His research interests include wireless communications and anti-jamming techniques.

Juan XU was born in Shaanxi, China, in 1980. She received the BS, MS and Ph.D degrees all in the School of Computer from Northwestern Polytechnical University (NPU), China, in 2002, 2005 and 2011, respectively. From 2011, she joined the School of Electronic and Control Engineering at Chang' an University, Xi'an, China. Her research interests include channel coding, OFDM transmission and spread spectrum systems. 